\title{
A family of transversely nonsimple knots
}

\author{
TIRASAN KHANDHAWIT \\ LENHARD NG
}

\begin{abstract}
We apply knot Floer homology to exhibit an infinite family of transversely nonsimple prime knots starting with $10_{132}$. We also discuss the combinatorial relationship between grid diagrams, braids and Legendrian and transverse knots in standard contact $\mathbb{R}^{3}$.
\end{abstract}

57R17; 57R58, 57M25

\section{Introduction}

Transverse knots play an important role in contact topology, but surprisingly little is known about their classification even in the simplest setting, $\mathbb{R}^{3}$ with the standard contact structure. Any transverse knot has an underlying topological knot type, and it also carries at least one other piece of data, the self-linking number. A topological knot type in $\mathbb{R}^{3}$ is transversely simple if transverse knots of that underlying type are completely classified by their self-linking number; otherwise, it is transversely nonsimple. Various knots are known to be transversely simple, including the unknot by Eliashberg [6], torus knots by Etnyre [8], and the figure eight knot by Etnyre and Honda [10].

It was only recently that some knot types were shown to be transversely nonsimple. Birman and Menasco [1] (see also their paper [2]) used braid theory to find a family of 3 -braids whose knot closures are transversely nonsimple; Etnyre and Honda [11] used contact-topological techniques to show that the $(2,3)$ cable of the $(2,3)$ torus knot is transversely nonsimple.

There has been much current effort to develop invariants of transverse knots that can be used to demonstrate transverse nonsimplicity. The first (and thus far only) invariant that has been shown to be effective lies, interestingly, not in contact-topological constructions like Symplectic Field Theory, but in knot Floer homology; see Ozsváth and Szabó [21] and Rasmussen [23]. The $\widehat{\theta}$ invariant in $\widehat{H F K}$ was introduced by Ozsváth, Szabó and Thurston [22] and was employed by the second author, Ozsváth and Szabó [16] to find several examples of transversely nonsimple knots, including $10_{132}$ and (a reproof 
of) the Etnyre-Honda cable example. Vértesi [24] used the examples of [16] and the behavior of $\hat{\theta}$ under connected sum to find infinite families of connected-sum examples of transversely nonsimple knots; see also Kawamuro [14]. Most recently, by studying the relationship between contact surgery and naturality properties of (a differently constructed version of) $\hat{\theta}$, Ozsváth and Stipsicz [20] proved transverse nonsimplicity for a wide family of two-bridge knots.

Absent the naturality techniques of [20], the applications of $\hat{\theta}$ to transverse simplicity have used a crude but surprisingly effective "vanishing criterion": if $T_{1}$ and $T_{2}$ are transverse knots and $\hat{\theta}\left(T_{1}\right)=0$ while $\hat{\theta}\left(T_{2}\right) \neq 0$, then $T_{1}$ and $T_{2}$ are distinct. The $\hat{\theta}$ invariant lies in the homology of a combinatorial chain complex introduced by Manolescu, Ozsváth and Sarkar [15], and [16] used a computer program to determine in examples whether $\hat{\theta}$ is null-homologous or not. However, reliance on a computer program obviously limits the number of transversely nonsimple examples that can be found.

In this paper, we find a two-parameter infinite family of prime, transversely nonsimple knots that can be distinguished using the vanishing criterion for $\hat{\theta}$. The idea is to find grid diagrams (the structures on which $\hat{\theta}$ is defined) where the computation of $\hat{\theta}$ is short enough to be carried out by hand. The resulting family of examples is not as simple in appearance as the two-bridge examples of [20], but has the advantage of needing only the combinatorial description of $\hat{\theta}$ and not an analysis of its image under contact surgery.

Our family is a generalization of the $10_{132}$ example from [16]. A transverse knot can be represented as a braid (see Section 2.3), and a fruitful technique for finding transversely nonsimple knots is to find braids that are related by a negative flype (cf [1]) and thus represent the same topological, but not necessarily transverse, knot. In correspondence with the second author, $\mathrm{H}$ Matsuda noted that the $10_{132}$ example can be expressed as a negative flype and proposed a one-parameter family of braids generalizing $10_{132}$. Here we expand Matsuda's conjectured family to a two-parameter family of braids related by a negative flype. ${ }^{1}$

Theorem 1.1 For any $a, b \geq 0$, the pair of 4-braids

$$
\sigma_{3} \sigma_{2}^{-2} \sigma_{3}^{2 a+2} \sigma_{2} \sigma_{3}^{-1} \sigma_{1}^{-1} \sigma_{2} \sigma_{1}^{2 b+2} \quad \text { and } \quad \sigma_{3} \sigma_{2}^{-2} \sigma_{3}^{2 a+2} \sigma_{2} \sigma_{3}^{-1} \sigma_{1}^{2 b+2} \sigma_{2} \sigma_{1}^{-1} \text {, }
$$

\footnotetext{
${ }^{1}$ We note in passing that the $7_{2}$ transverse knots in [16, Figure 11], shown to be distinct in [20], are also related by a negative flype, $\sigma_{3}^{2} \sigma_{2}^{2} \sigma_{3}^{-1} \sigma_{1}^{2} \sigma_{2} \sigma_{1}^{-1} \leftrightarrow \sigma_{3}^{2} \sigma_{2}^{2} \sigma_{3}^{-1} \sigma_{1}^{-1} \sigma_{2} \sigma_{1}^{2}$, as can be checked using the techniques from this paper.
} 
related by a negative flype and thus representing the same topological knot and having the same self-linking number, represent distinct transverse knots. In particular, the topological knot types given by these pairs, which are prime, are transversely nonsimple.

The $10_{132}$ case is $a=b=0$; in this case, the braids in Theorem 1.1 are transversely isotopic to $L_{1}$ and $L_{2}$, respectively, from [22, Section 3.1]. Other small knots in this family, with the corresponding values of $(a, b)$, include $(0,1)=12_{120}^{n},(1,0)=12_{199}^{n}$, $(0,2)=14_{2016}^{n},(1,1)=14_{3606}^{n}$ and $(2,0)=14_{5045}^{n}$, where the identifications in the Dowker-Thistlethwaite knot enumeration come from Knotscape [12].

A Shumakovitch has noticed that some (perhaps all) of this family of knots have interesting odd Khovanov homology [19]. More precisely, the six examples listed above have the unusual feature that their odd Khovanov homology completely vanishes in homological degree 0 . We do not know if this is a coincidence.

We believe that this two-parameter family is in fact part of a four-parameter family of transversely nonsimple knots given by the closures of the braids

$$
\sigma_{3} \sigma_{2}^{-2 c-2} \sigma_{3}^{2 a+2} \sigma_{2} \sigma_{3}^{-2 d-1} \sigma_{1}^{-1} \sigma_{2} \sigma_{1}^{2 b+2}
$$

for $a, b, c, d \geq 0$. We have checked several examples using the computer program of [16] but do not have a general proof for the case $(c, d) \neq(0,0)$.

In order to apply the $\hat{\theta}$ invariant to braids to prove Theorem 1.1, we need techniques for translating between braids, grid diagrams and Legendrian and transverse knots in standard contact $\mathbb{R}^{3}$. These translations are presented in Section 2 and are by now well-known to experts, but we were unable to find any full write-ups in the literature. In particular, the algorithms for obtaining a Legendrian knot from a braid and a braid from a grid diagram may be of independent interest. We then prove Theorem 1.1 in Section 3.

Acknowledgments We would like to thank H Matsuda, A Shumakovitch and D Thurston for useful discussions. Part of this work also appeared in the first author's undergraduate honors thesis at Duke University. The second author is supported by NSF grant DMS-0706777.

\section{Braids, grid diagrams and transverse Knots}

Here we review several different approaches to transverse knots in standard contact $\mathbb{R}^{3}$. Most of the material in this section can be found in the Etnyre survey [9] or, in the case of grid diagrams, [22]. The new content consists of results in Section 2.4 giving methods to translate between grid diagrams, braids, and Legendrian knots, but even these are "folk theorems" that have been floating around the subject for several years. 


\subsection{Legendrian and transverse knots}

Let

$$
\xi_{\text {std }}=\operatorname{ker}(d z-y d x)
$$

denote the standard contact structure on $\mathbb{R}^{3}$. A Legendrian knot in $\left(\mathbb{R}^{3}, \xi_{\text {std }}\right)$ is an oriented knot that is everywhere tangent to $\xi_{\text {std }}$. A transverse knot is an oriented knot that is everywhere transverse to $\xi_{\text {std }}$, with the orientation agreeing with the usual coorientation on $\xi_{\text {std }}$; that is, $d z-y d x>0$ along the orientation of a transverse knot. Any smooth knot in $\mathbb{R}^{3}$ can be $C^{0}$ perturbed to both Legendrian and transverse knots. We consider Legendrian and transverse knots up to Legendrian and transverse isotopy, isotopy through Legendrian and transverse knots, respectively.

There is a many-to-one correspondence between Legendrian and transverse knots. Any Legendrian knot $L$ can be perturbed to a transverse knot $L_{+}$, the positive pushoff of $L$, by pushing each point on $L$ in a direction transverse to the contact plane; the positive pushoff is unique up to transverse isotopy. There is an "inverse" operation that perturbs any transverse knot to a Legendrian knot, but this is only well-defined up to Legendrian isotopy and negative stabilization/destabilization of Legendrian knots (see below). Thus one can view transverse knots up to transverse isotopy as Legendrian knots up to Legendrian isotopy and negative de/stabilization.

A convenient way to depict Legendrian and transverse knots is through their front projections to the $x z$ plane. The front projection of a generic Legendrian knot has no vertical tangencies and has only double points and semicubical cusps as singularities. At each double point, the arc of more negative slope passes over the arc of more positive slope. Any front of this type is the front projection of a Legendrian knot.
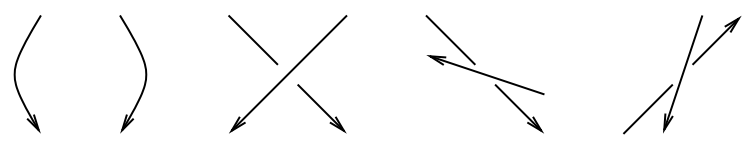

Figure 1: Forbidden segments in the front projection of a transverse knot

On the other hand, the front projection of a generic transverse knot is a standard knot diagram, with only double points as singularities, but with two restrictions: any point in the projection with a vertical tangency must be oriented upwards, and at a crossing, we cannot simultaneously have the overcrossing arc pointing to the left, the undercrossing arc pointing to the right, and the overcrossing arc of greater slope than the undercrossing arc. See Figure 1. Any knot projection without these forbidden segments is the front projection of a transverse knot, unique up to transverse isotopy, and two transverse 


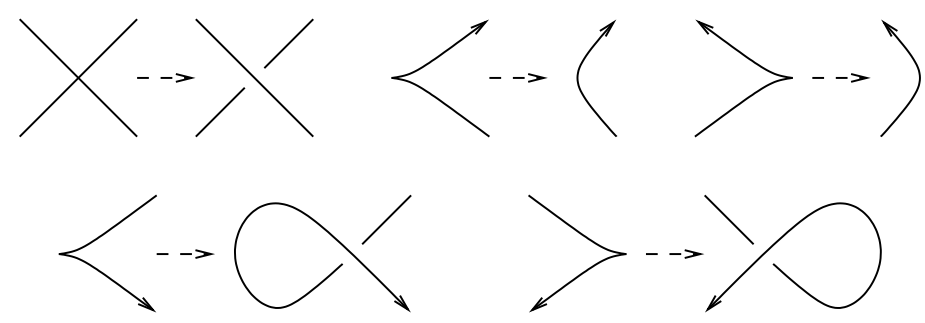

Figure 2: Obtaining the front of the transverse pushoff from a Legendrian front

knots are transversely isotopic if and only if their front projections are isotopic through diagrams that do not contain any forbidden segments.

With front projections, it is easy to see the correspondence between Legendrian and transverse knots. The front of a Legendrian knot can be turned into the front of its positive transverse pushoff by smoothing out upward-pointing cusps and replacing downward-pointing cusps by loops; see Figure 2. In a related vein, we define the positive and negative stabilizations $S_{ \pm}(L)$ of a Legendrian knot $L$ to be the Legendrian knots whose fronts are obtained from the front of $L$ by adding in a zigzag whose cusps point downward or upward; see Figure 3. Both stabilizations are well-defined up to Legendrian isotopy. It is clear from the front picture that a Legendrian knot and its negative stabilization have positive transverse pushoffs that are transversely isotopic. A result due in the $\mathbb{R}^{3}$ case to [7] states that two Legendrian knots are related by Legendrian isotopy and negative de/stabilization if and only if their positive transverse pushoffs are related by transverse isotopy.

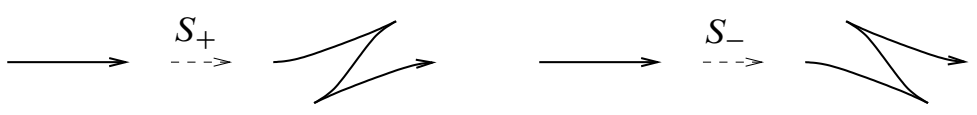

Figure 3: Positive and negative stabilizations of a Legendrian front

\subsection{Grid diagrams}

Closely related to front projections is another fruitful method of representing Legendrian knots, via grid diagrams. A grid diagram is an $n \times n$ square grid with a collection of $n$ $X$ 's and $n O$ 's in the grid, such that each row or column contains exactly one $X$ and one $O$, and no square in the grid contains both an $X$ and an $O$.

One obtains a knot (or link) diagram from a grid diagram by connecting $O$ 's to $X$ 's horizontally, connecting $X$ 's to $O$ 's vertically, and stipulating that horizontal segments always pass over vertical segments whenever they cross. (Note that this is the opposite 
of the standard convention for grid diagrams.) In this way, any knot can be represented by a grid diagram. Indeed, we can view a grid diagram $G$ as (the front of) a Legendrian knot $L(G)$ by turning it $45^{\circ}$ clockwise, smoothing upward- and downward-pointing corners, and turning leftward- and rightward-pointing corners into cusps. See Figure 7 below. Any Legendrian knot is Legendrian isotopic to a knot obtained in this way from some grid diagram.

There is a sequence of elementary moves on grid diagrams, the Cromwell moves [4], that relate any two grid diagrams that represent topologically isotopic knots: torus translation, commutation, and stabilization/destabilization. The stabilization moves divide further into (essentially) four types, labeled $X: N W, X: N E, X: S W$, and $X: S E$ in the notation of [22]. Of the Cromwell moves, torus translation, commutation, and $X: N W$ and $X: S E$ de/stabilization preserve Legendrian isotopy type, while $X: N E$ (resp. $X: S W$ ) stabilization is positive (resp. negative) stabilization in the Legendrian category.

\subsection{Braids and transverse knots}

In some sense, the role played by grid diagrams for Legendrian knots is played by braids for transverse knots. Let

$$
\xi_{\text {rot }}=\operatorname{ker}(d z-y d x+x d y)
$$

denote the rotationally symmetric tight contact structure on $\mathbb{R}^{3}$. There is an orientationpreserving diffeomorphism $\phi$ of $\mathbb{R}^{3}$, given by $\phi(x, y, z)=(x, 2 y, x y+z)$, that sends $\xi_{\text {rot }}$ to $\xi_{\text {std }}$. We can define transverse knots for $\xi_{\text {rot }}$ in the same way as transverse knots for $\xi_{\text {std }}$, and $\phi$ sends a knot transverse to $\xi_{\text {rot }}$ to a knot transverse to $\xi_{\text {std }}$. Thus we can view any knot transverse to $\xi_{\text {rot }}$ as a transverse knot in the sense of Section 2.1.

The closed curve $\{(\cos t, \sin t, 0) \mid 0 \leq t \leq 2 \pi\}$ traces out an unknot $T_{0}$ transverse to $\xi_{\text {rot }}$. We can then view any braid $B$ as a transverse knot as follows. Embed the closure of $B$ in a solid torus, and embed this solid torus as a small tubular neighborhood of $T_{0}$. The braid then becomes a knot (or link) $T(B)$ in $\mathbb{R}^{3}$ transverse to $\xi_{\text {rot }}$, and can be mapped to a transverse $\operatorname{knot} \phi(T(B))$ in $\left(\mathbb{R}^{3}, \xi_{\text {std }}\right)$ via the contactomorphism $\phi$.

Braids that are conjugate in the braid group yield transversely isotopic knots. More interestingly, let a positive braid stabilization be the operation that replaces a braid $B \in B_{n}$ by $B \sigma_{n} \in B_{n+1}$. Then we have the following result.

Proposition 2.1 (Transverse Markov Theorem [18; 25]) Let $B_{1}, B_{2}$ be braids. Then $T\left(B_{1}\right), T\left(B_{2}\right)$ are transversely isotopic in $\left(\mathbb{R}^{3}, \xi_{\text {rot }}\right)$ if and only if $B_{1}, B_{2}$ are related by a sequence of braid conjugations and positive braid stabilizations and destabilizations. 


\subsection{Translating between the three pictures}

Given a braid word $B$, one can create the front of a Legendrian knot $L(B)$ in a natural way as shown in Figure 4, cf [13]. Draw the braid from left to right; each positive crossing becomes part of the front in the obvious way, while each negative crossing is represented by a zigzag and crossing in the front. The corresponding left and right ends of the braid are then connected through arcs with one left cusp and one right cusp apiece.
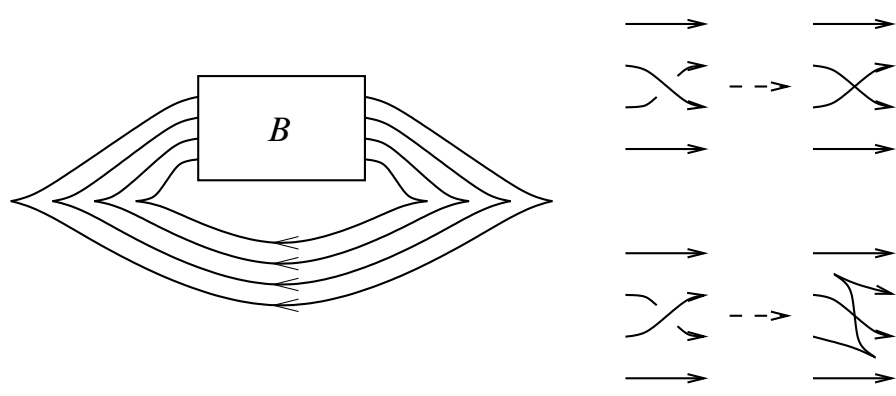

Figure 4: The Legendrian front $L(B)$ obtained from a braid word $B$

We note that $L(B)$ is associated to a braid word and not a braid isotopy class. If $B$ changes by braid isotopy, $L(B)$ changes by a combination of Legendrian isotopies and negative Legendrian de/stabilizations: replacing $\sigma_{i} \sigma_{i+1} \sigma_{i}$ by $\sigma_{i+1} \sigma_{i} \sigma_{i+1}$ preserves Legendrian isotopy type, while introducing $\sigma_{i} \sigma_{i}^{-1}$ or $\sigma_{i}^{-1} \sigma_{i}$ corresponds to one negative stabilization. Thus, for $B$ a braid, $L(B)$ is only well-defined up to negative Legendrian de/stabilization; however, the positive transverse pushoff $L(B)_{+}$ constitutes a well-defined transverse isotopy class.

In addition, it is straightforward to check that changing $B$ by positive braid stabilization preserves the Legendrian isotopy, while conjugating $B$ in the braid group changes $L(B)$ at most by negative Legendrian de/stabilization (for the latter, see also the appendix on the Legendrian satellite construction in [17]). We conclude by the Transverse Markov Theorem that if two braids $B_{1}, B_{2}$ have transversely isotopic knot closures in ( $\mathbb{R}^{3}, \xi_{\text {rot }}$ ), then the positive transverse pushoffs $L\left(B_{1}\right)_{+}, L\left(B_{2}\right)_{+}$are transversely isotopic knots in $\left(\mathbb{R}^{3}, \xi_{\text {std }}\right)$. In fact, any braid $B$, viewed as a transverse knot in $\left(\mathbb{R}^{3}, \xi_{\text {rot }}\right)$, is the same as $L(B)_{+}$, viewed as a transverse knot in $\left(\mathbb{R}^{3}, \xi_{\text {std }}\right)$.

Proposition 2.2 Let $\phi$ be the contactomorphism between $\left(\mathbb{R}^{3}, \xi_{\text {rot }}\right)$ and $\left(\mathbb{R}^{3}, \xi_{\text {std }}\right)$ from Section 2.3. If $B$ is a braid and $T(B)$ is the transverse knot in $\left(\mathbb{R}^{3}, \xi_{\text {rot }}\right)$ corresponding to $B$, then $\phi(T(B))$ and $L(B)_{+}$are transversely isotopic knots in $\left(\mathbb{R}^{3}, \xi_{\text {std }}\right)$. 

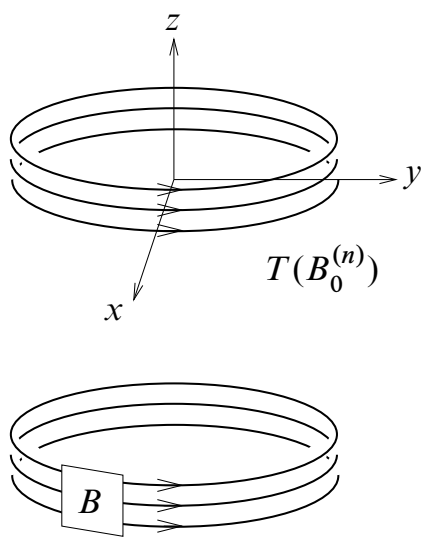

$T(B)$
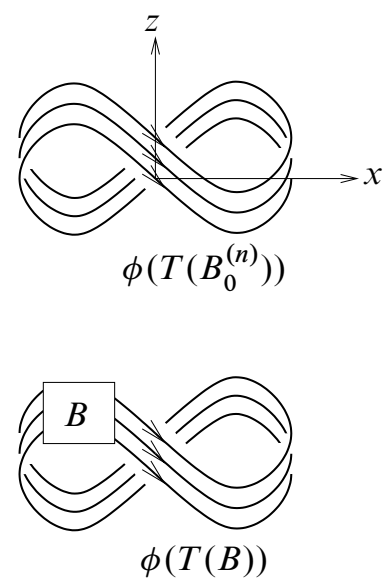

Figure 5: Transverse knots $T\left(B_{0}^{(n)}\right), T(B)$ in $\xi_{\text {rot }}$ and the corresponding fronts of transverse knots in $\xi_{\text {std }}$

Proof Under $\phi$, the standard transverse unknot $T_{0}$ in $\xi_{\text {rot }}$ maps to an unknot whose front projection is a figure 8 . For any $n$, view the trivial $n$-component braid $B_{0}^{(n)}$ as the transverse link in $\xi_{\text {rot }}$ defined by

$$
T\left(B_{0}^{(n)}\right)=\{(\cos t, \sin t, k \epsilon) \mid 0 \leq t \leq 2 \pi, k=0, \ldots, n-1\}
$$

for small $\epsilon>0$. The front projection of $\phi\left(T\left(B_{0}^{(n)}\right)\right)$ is a collection of $n$ figure 8's that differ by $\epsilon$ translations in the $z$ direction; see Figure 5 .

Let $B$ be a braid with $n$ strands. In cylindrical coordinates $(r, \theta, z)$ on $\mathbb{R}^{3}$, we can choose $T(B)$ to agree with $T\left(B_{0}^{(n)}\right)$ except in a neighborhood of $\theta=5 \pi / 4$, where the entire braid $B$ lives. Then the front of $\phi(T(B))$ agrees with $\phi\left(T\left(B_{0}^{(n)}\right)\right)$ except in the braiding region near $(x, z)=(-1 / \sqrt{2}, 1 / 2)$.

In the braiding region for the front of $\phi(T(B))$, we can draw $B$ in the standard way, such that each strand goes from left to right without vertical tangencies. We can then modify the front of $\phi(T(B))$ by a transverse isotopy so that the $n$ figure 8 's do not intersect anywhere outside of the braiding region. The result is a transverse front that is transversely isotopic to the front of $L(B)_{+}$; see Figure 6 . The result follows.

We next turn to the relation between grid diagrams and braids. Given any grid diagram $G$, one can construct a braid $B(G)$ as follows (cf $[4 ; 5 ; 16]$ ). Connect $O$ 's to $X$ 's horizontally as usual. When an $O$ lies vertically above an $X$, connect them; when an $O$ lies vertically under an $X$, draw two vertical line segments, one from the $O$ down to the bottom of the grid diagram, one from the $X$ up to the top of the grid 

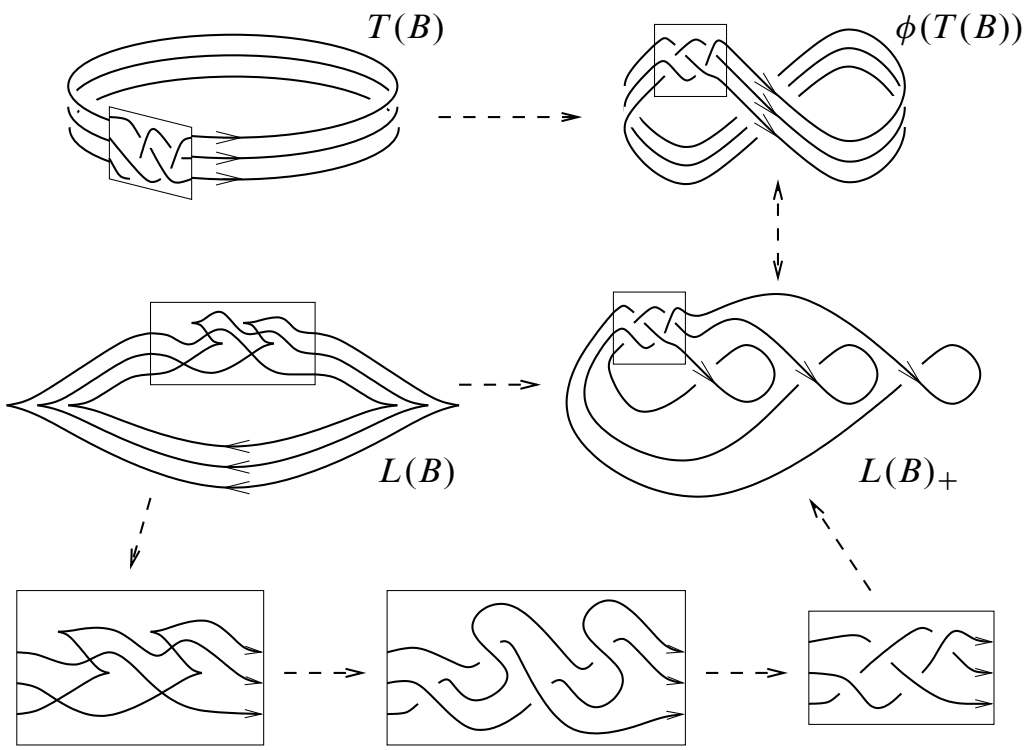

Figure 6: Transverse isotopy between $\phi(T(B))$ and $L(B)_{+}$. The top row is as in Figure 5. The middle row shows the positive transverse pushoff of $L(B)$, resulting in a transverse front that is isotopic to the front for $\phi(T(B))$. The bottom row shows a detail of the braiding region for the fronts of $L(B)$; $L(B)_{+}$; and $L(B)_{+}$after a transverse isotopy.

diagram. Whenever two line segments cross, have the horizontal segment cross over the vertical segment as before. We can now orient all segments so that $O$ 's point to $X$ 's horizontally as usual, and all vertical segments are oriented upwards. The result can be viewed as a braid from the bottom of the grid diagram to the top. See Figure 7. We remark that the closure of $B(G)$ is isotopic to the knot given by $G$, and that any braid is $B(G)$ for some grid diagram $G$.

To a grid diagram $G$, we have now associated a Legendrian knot $L(G)$ and a braid $B(G)$. The following result is a compatibility result for these two constructions as well as the construction $L(B)$.

Proposition 2.3 If $G$ is a grid diagram, then $L(G)_{+}$and $L(B(G))_{+}$are transversely isotopic; that is, the Legendrian knots $L(G)$ and $L(B(G))$ are related by Legendrian isotopy and negative de/stabilization.

Proof Let $G$ be an $n \times n$ grid diagram, and suppose $B(G)$ has $m$ strands. One can associate to $B(G)$ a natural $(n+2 m) \times(n+2 m)$ grid diagram $G^{\prime}$ within which the 


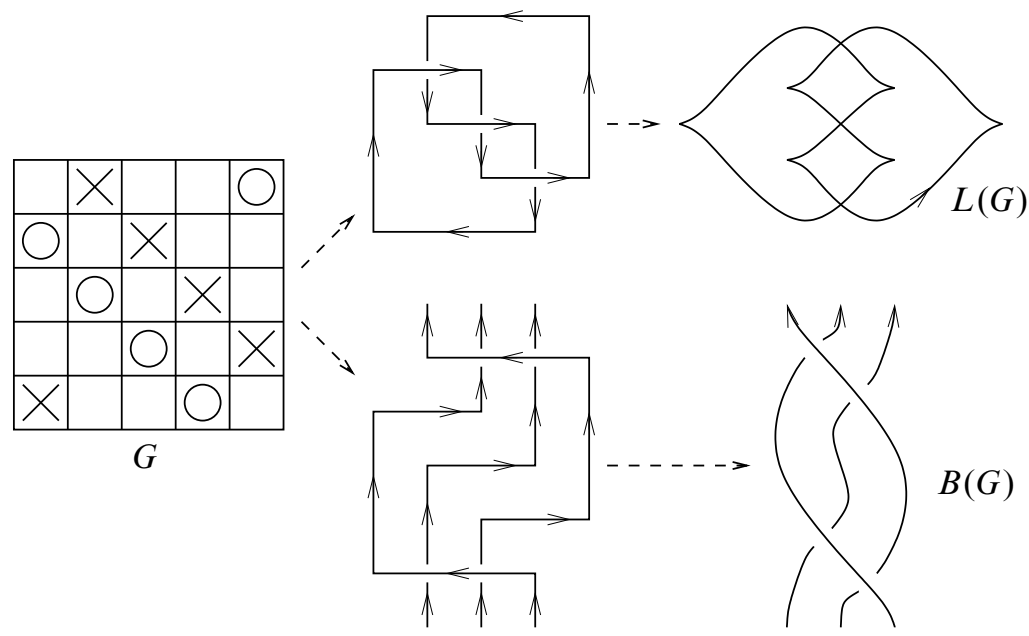

Figure 7: Constructing a Legendrian knot $L(G)$ and a braid $B(G)$ from a grid diagram $G$. In this example, we read $B(G)$ bottom to top to get $B(G)=$ $\sigma_{1}^{-1} \sigma_{2}^{-1} \sigma_{1}^{-1} \sigma_{2}^{-1}$.

original diagram $G$ appears, such that $L(B(G))$ is Legendrian isotopic to $S_{-}^{k}\left(L\left(G^{\prime}\right)\right)$ for some $k$; see Figure 8. (More precisely, $k$ is the number of appearances of subwords of the form $\sigma_{i}^{-1} \sigma_{i+1}^{-1}$ in $B(G)$.) There are $m$ "braid" parts of the new grid diagram, each of which begins at an $X$, goes up out of $G$, curves around $G$ to the right, and ends at an $O$. We can eliminate each of these parts in succession, via grid commutation and two grid destabilizations, one a Legendrian isotopy and one a negative Legendrian destabilization; see Figure 9 for an illustration. The end result is $L(G)$, whence $L(B(G))$ is Legendrian isotopic to $S_{-}^{m+k}(L(G))$, and the proposition follows.

We remark that one can obtain another braid $B^{\prime}(G)$ from a grid diagram $G$ such that Proposition 2.3 also holds. Instead of forcing all vertical segments to point upwards, we instead force all horizontal segments to point leftwards. This yields a braid $B^{\prime}(G)$ by reading from right to left. The two braids $B(G)$ and $B^{\prime}(G)$ are almost never identical or even conjugate, but they do represent the same transverse knot.

To see this, define the diagonal mirror $G^{\prime}$ of a grid diagram $G$ to be the grid diagram obtained by reflecting in the main (upper left to lower right) diagonal and interchanging all $X$ 's and $O$ 's. The diagonal mirror represents the same topological knot as the original grid diagram, and in fact represents the same Legendrian knot up to Legendrian isotopy. This follows from the fact that the diagonal mirror replaces a Legendrian front in the $x z$ plane with its reflection in the $z$ axis, corresponding to the contactomorphism $(x, y, z) \mapsto(-x,-y, z)$ of $\left(\mathbb{R}^{3}, \xi_{\text {std }}\right)$, which is just a rotation in the $x y$ plane and 


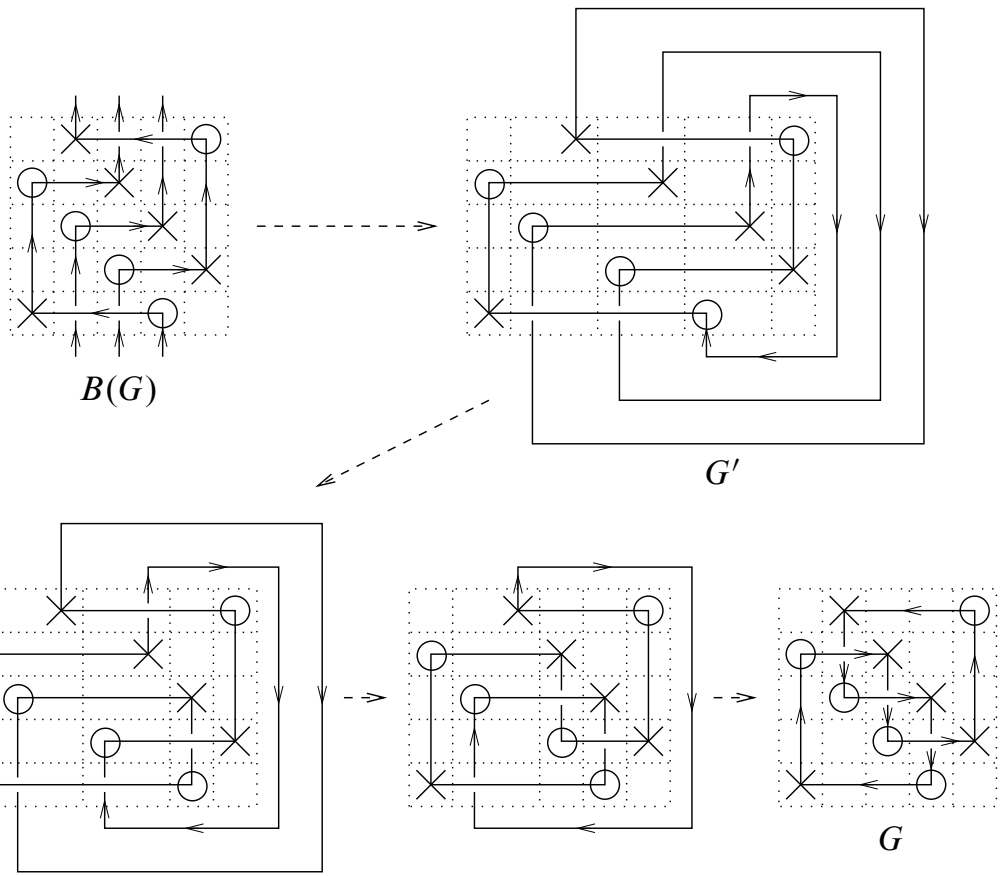

Figure 8: Destabilizing a grid diagram $G^{\prime}$ obtained from $B(G)$ to recover $G$. The diagram $G^{\prime}$, viewed as a Legendrian knot, is itself some number of negative destabilizations of $L(B(G))$ ( 2 in this case).

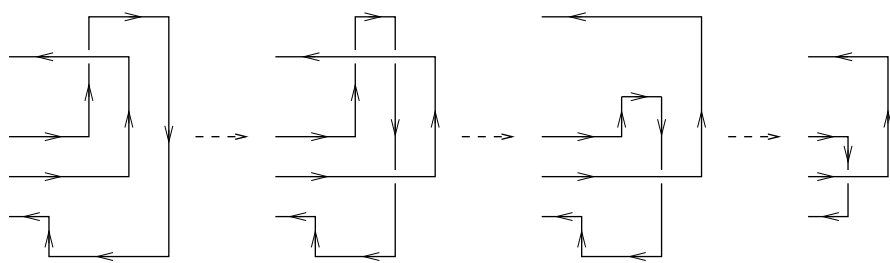

Figure 9: Decomposing the diagonal dashed arrow in Figure 8 into a composition of commutations and destabilizations. The first two steps are commutations; the final step is two grid destabilizations, both preserving transverse type.

preserves Legendrian knots up to isotopy. Now $B^{\prime}(G)$ is the same braid as $B\left(G^{\prime}\right)$ and thus corresponds to the same transverse knot as $B(G)$.

We summarize the results from this section in the following diagram, where $G, B$, $L$, and $T$ represent grid diagrams, braids, Legendrian knots/links in $\left(\mathbb{R}^{3}, \xi_{\text {std }}\right)$, and 
transverse knots/links in $\left(\mathbb{R}^{3}, \xi_{\text {std }}\right)$ respectively.

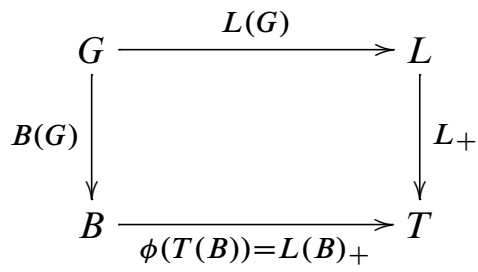

The equality at the bottom of this diagram is Proposition 2.2, while Proposition 2.3 states that the square commutes.

\section{Proof of Theorem 1.1}

For $a, b \geq 0$, we write the two relevant 4 -braids as

$$
\begin{aligned}
& B_{1}(a, b)=\sigma_{3} \sigma_{2}^{-2} \sigma_{3}^{2 a+2} \sigma_{2} \sigma_{3}^{-1} \sigma_{1}^{-1} \sigma_{2} \sigma_{1}^{2 b+2} \\
& B_{2}(a, b)=\sigma_{3} \sigma_{2}^{-2} \sigma_{3}^{2 a+2} \sigma_{2} \sigma_{3}^{-1} \sigma_{1}^{2 b+2} \sigma_{2} \sigma_{1}^{-1} .
\end{aligned}
$$

We now break the proof of Theorem 1.1 into several parts.

Proposition 3.1 The knot given by the closure of $B_{1}(a, b)$ (or $\left.B_{2}(a, b)\right)$ is prime.

Proof Let $K(a, b)$ denote the closure of $B_{1}(a, b)$. Since braid index minus 1 is additive under connected sum, if $K(a, b)$ is composite, then one of its direct summands must be a knot of braid index 2 , ie, some torus knot $T(2,2 n+1)$ for some integer $n \neq 0,-1$. It thus suffices to show that for any $a, b \geq 0$ and $n \neq 0,-1, P(T(2,2 n+1))(x, z)$ does not divide $P(K(a, b))(x, z)$, where $P(K)(x, z)$ is the HOMFLY-PT polynomial of $K$, defined as usual by $P(\bigcirc)=1, x P(\lambda)-x^{-1} P(\searrow)=z P(こ)$.

It is straightforward to check, by induction and the HOMFLY-PT skein relation, that

$$
\begin{aligned}
P(K(a, b))(x, 0)= & x^{-2 a-2 b-6}\left(-2 a-2+(3 a+3-b) x^{2}+(b-a) x^{4}\right) \\
P(T(2,2 n+1))(x, 0)= & x^{-2 n-2}\left(-n+(n+1) x^{2}\right) \\
P(K(a, b))(x, 2 i)= & -\left(-x^{2}\right)^{-a-b-3}(2(1+a)(1+2 b) \\
& \left.+(3+3 a+7 b+8 a b) x^{2}+(a+3 b+4 a b) x^{4}\right) \\
P(T(2,2 n+1))(x, 2 i)= & -\left(-x^{2}\right)^{-n-1}\left(n+(n+1) x^{2}\right),
\end{aligned}
$$

where $i=\sqrt{-1}$. If $P(T(2,2 n+1))$ divides $P(K(a, b))$, then the first two equations readily imply that $2 a+2+(a+b+1) n=n^{2}$, while the last two equations imply that $2 a+2+4 b+4 a b+(a+b+1) n=n^{2}$. It follows that $b=0$ and $(a+1)(n+2)=n^{2}$. 
Since $n+2$ divides $n^{2}$, we have $|n+2|=\left|\operatorname{gcd}\left(n+2, n^{2}\right)\right| \leq(\operatorname{gcd}(n+2, n))^{2} \leq 4$, and a quick check shows that $n$ must be one of $-1,0,2$. Thus $n=2$ and $a=0$; but $K(0,0)$ is (the mirror of) $10_{132}$ and hence prime.

To apply the $\hat{\theta}$ invariant to $B_{1}(a, b)$ and $B_{2}(a, b)$, we need grid diagrams for both braids. It is possible to create grid diagrams directly from the braids, but to facilitate the computation we need particular diagrams.

Proposition 3.2 Let $G_{1}(a, b)$ and $G_{2}(a, b)$ be the $(2 a+2 b+9) \times(2 a+2 b+9)$ and $(2 a+2 b+10) \times(2 a+2 b+10)$ grid diagrams depicted in Figures 10 and 11 . Then $B\left(G_{1}(a, b)\right)$ and $B\left(G_{2}(a, b)\right)$ represent the same transverse knots as $B_{1}(a, b)$ and $B_{2}(a, b)$, respectively.

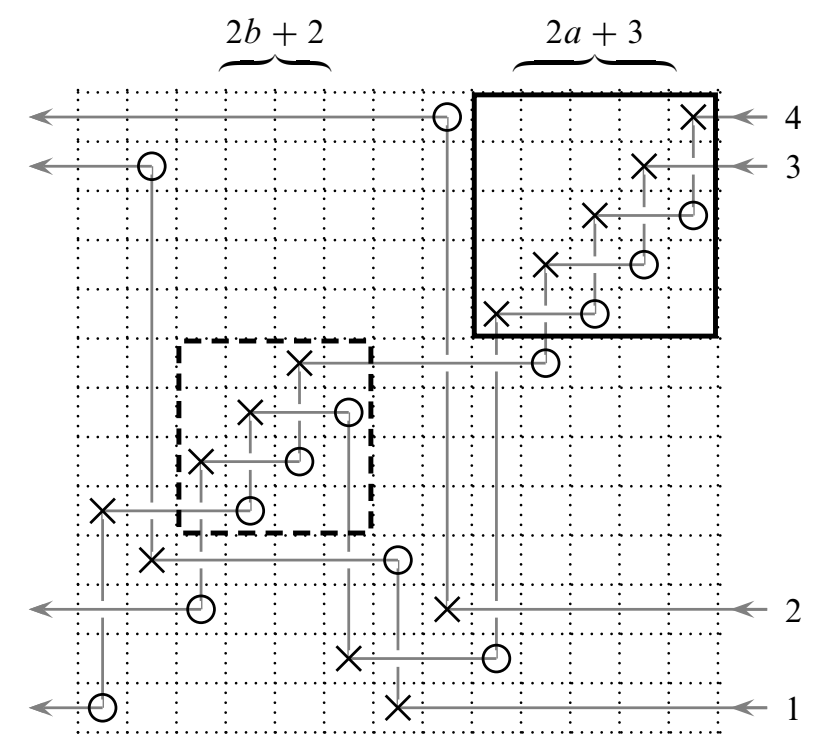

Figure 10: The grid diagram $G_{1}(a, b)$ for $B_{1}(a, b)$, along with the braid $B^{\prime}\left(G_{1}(a, b)\right)$ with strands numbered. The solid box denotes a pattern consisting of $X$ 's on the diagonal and $O$ 's on the second subdiagonal; the dashed box has $X$ 's on the first superdiagonal and $O$ 's on the first subdiagonal.

Proof The braids $B\left(G_{1}(a, b)\right)$ and $B\left(G_{2}(a, b)\right)$ have a large number of strands. It is easier to work with $B^{\prime}\left(G_{1}(a, b)\right)$ and $B^{\prime}\left(G_{2}(a, b)\right)$, where $B^{\prime}$ is the braid constructed by stipulating that all horizontal segments point leftward; see Section 2.4. It was shown in Section 2.4 that $B(G)$ and $B^{\prime}(G)$ represent the same transverse knot for any grid diagram $G$. 


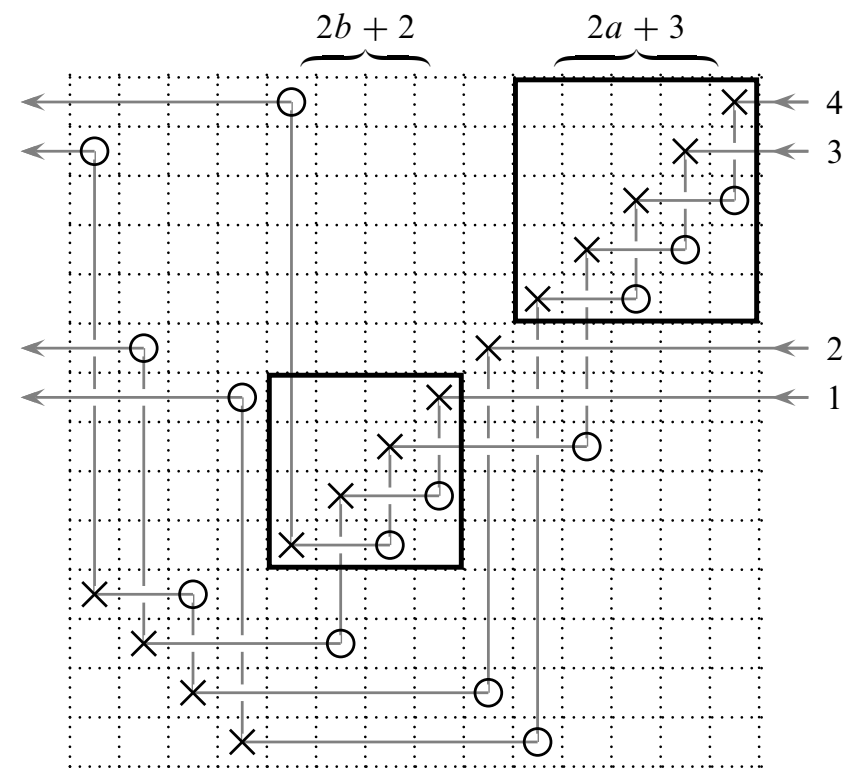

Figure 11: The grid diagram $G_{2}(a, b)$ for $B_{2}(a, b)$, along with the braid $B^{\prime}\left(G_{2}(a, b)\right)$

We readily calculate from the grid diagrams that $B^{\prime}\left(G_{1}(a, b)\right)$ and $B^{\prime}\left(G_{2}(a, b)\right)$ are 4-braids given by

$$
\begin{aligned}
& B^{\prime}\left(G_{1}(a, b)\right)=\sigma_{3}^{2 a+3} \sigma_{2} \sigma_{3}^{-1} \sigma_{1}^{-2} \sigma_{2}^{2 b+1} \sigma_{1} \sigma_{2}^{-1} \sigma_{1} \\
& B^{\prime}\left(G_{2}(a, b)\right)=\sigma_{3}^{2 a+2} \sigma_{2} \sigma_{1} \sigma_{3} \sigma_{2} \sigma_{1} \sigma_{3} \sigma_{2} \sigma_{3}^{2 b+1} \sigma_{1}^{-1} \sigma_{2}^{-1} \sigma_{1}^{-2} \sigma_{2}^{-1} \sigma_{1}^{-1} \sigma_{2}^{-1} .
\end{aligned}
$$

From relations in the braid group, we find that

$$
B^{\prime}\left(G_{2}(a, b)\right)=\sigma_{3}^{2 a+2} \sigma_{2} \sigma_{3}^{-1} \sigma_{1}^{2 b+2} \sigma_{2} \sigma_{1}^{-1} \sigma_{3} \sigma_{2}^{-2}
$$

and thus $B^{\prime}\left(G_{2}(a, b)\right)$ is conjugate to $B_{2}(a, b)$.

The braids $B_{1}(a, b)$ and $B^{\prime}\left(G_{1}(a, b)\right)$ are not conjugate, but are related by conjugation and exchange moves. For our purposes, we recognize two exchange moves on 4-braids, related by conjugation:

- $\sigma_{1}$ exchange: $b_{1} \sigma_{1}^{-1} b_{2} \sigma_{1} b_{3} \leftrightarrow b_{1} \sigma_{1} b_{2} \sigma_{1}^{-1} b_{3}$, where $b_{1}, b_{2}, b_{3}$ are braids in the subgroup generated by $\sigma_{2}, \sigma_{3}$

- $\sigma_{3}$ exchange: $b_{1} \sigma_{3}^{-1} b_{2} \sigma_{3} b_{3} \leftrightarrow b_{1} \sigma_{3} b_{2} \sigma_{3}^{-1} b_{3}$, where $b_{1}, b_{2}, b_{3}$ are braids in the subgroup generated by $\sigma_{1}, \sigma_{2}$.

Since an exchange move is a composition of conjugations, one positive braid stabilization, and one positive destabilization, it does not change the transverse type of the 
braid; see [3, Figure 8]. Now we have

$$
\begin{aligned}
B^{\prime}\left(G_{1}(a, b)\right) \stackrel{\operatorname{conj} \sigma_{1}}{\longrightarrow} & \sigma_{1} \sigma_{3}^{2 a+3} \sigma_{2} \sigma_{3}^{-1} \sigma_{1}^{-2} \sigma_{2}^{2 b+1} \sigma_{1} \sigma_{2}^{-1} \\
& =\sigma_{2}^{-1} \sigma_{3}^{-1} \sigma_{2}^{2 a+3} \sigma_{1} \sigma_{2}^{-1} \sigma_{3} \sigma_{2} \sigma_{1}^{-1} \sigma_{2}^{2 b+1} \sigma_{1} \sigma_{2}^{-1} \\
\stackrel{\operatorname{exch} \sigma_{3}}{\longrightarrow} & \sigma_{2}^{-1} \sigma_{3} \sigma_{2}^{2 a+3} \sigma_{1} \sigma_{2}^{-1} \sigma_{3}^{-1} \sigma_{2} \sigma_{1}^{-1} \sigma_{2}^{2 b+1} \sigma_{1} \sigma_{2}^{-1} \\
& =\sigma_{2}^{-1} \sigma_{3} \sigma_{2}^{2 a+3} \sigma_{3} \sigma_{2}^{-1} \sigma_{1}^{-1} \sigma_{2} \sigma_{3}^{-1} \sigma_{2}^{2 b+1} \sigma_{1} \sigma_{2}^{-1} \\
& \stackrel{\operatorname{exch} \sigma_{1}}{\longrightarrow} \sigma_{2}^{-1} \sigma_{3} \sigma_{2}^{2 a+3} \sigma_{3} \sigma_{2}^{-1} \sigma_{1} \sigma_{2} \sigma_{3}^{-1} \sigma_{2}^{2 b+1} \sigma_{1}^{-1} \sigma_{2}^{-1} \\
& \stackrel{\operatorname{conj} \sigma_{3}}{\longrightarrow} B_{1}(a, b),
\end{aligned}
$$

where "conj $\sigma_{k}$ " is conjugation by $\sigma_{k}, B \mapsto \sigma_{k} B \sigma_{k}^{-1}$.

We now use $\hat{\theta}$ to show that $G_{1}(a, b)$ and $G_{2}(a, b)$ are of different transverse types; more precisely, if we define transverse knots

$$
T_{i}(a, b)=L\left(G_{i}(a, b)\right)_{+}=\phi\left(T\left(B_{i}(a, b)\right)\right)
$$

in $\left(\mathbb{R}^{3}, \xi_{\text {std }}\right)$ for $i=1,2$, then $T_{1}(a, b)$ and $T_{2}(a, b)$ are not transversely isotopic. This will complete the proof of Theorem 1.1.

Recall from [22] that if $T$ is a transverse knot, then $\hat{\theta}(T)$ is an element of the knot Floer homology $\widehat{H F K}(m(T))$, where $m(T)$ is the topological mirror of $T$. If $T=L(G)_{+}$ for a grid diagram $G$, then $\widehat{H F K}(m(T))$ can be combinatorially computed from $G$ as in [15]. It is easier to consider a variant $\widetilde{H F K}(m(T))$ of $\widehat{H F K}(m(T))$, in which there is a corresponding element $\tilde{\theta}(T)\left(=j_{*}(\hat{\theta})\right.$ in [16]); then $\tilde{\theta}(T)=0$ if and only if $\hat{\theta}(T)=0$ [16, Section 4].

We assume some familiarity with the combinatorial definition of $\widetilde{H F K}$ over $\mathbb{Z} / 2$ from [15] (or [16;22]). If $T=L(G)_{+}$for an $n \times n$ grid diagram $G$, then the complex $\widetilde{C F K}(m(T))$ is generated by $n$ ! states labeled by permutations of $\{1, \ldots, n\}$. A state $(\pi(1), \ldots, \pi(n))$ can be depicted in the grid as a set of $n$ points $\{(i, \pi(i))\}$, where $(i, j)$ is the intersection of vertical line $i$ and horizontal line $j$; here the vertical (resp. horizontal) lines are numbered left to right (resp. bottom to top) starting with 1 . The differential $\partial$ on $\widetilde{C F K}(m(T))$ is represented pictorially by

$$
\partial(\square)=\sum \square
$$

where the sum is over all rectangles not containing any $X$ 's, $O$ 's, or other points in the state. If $\partial(y)$ contains $x$ as a term, then we write $x \leftarrow y$ and $y \rightarrow x$. The transverse invariant $\tilde{\theta}$ is the image in $\widetilde{H F K}(m(T))$ of the state $x^{+}$given by the upper right corners of the $X$ 's. 
Proposition 3.3 We have $\tilde{\theta}\left(T_{1}(a, b)\right)=0$ and hence $\hat{\theta}\left(T_{1}(a, b)\right)=0$.

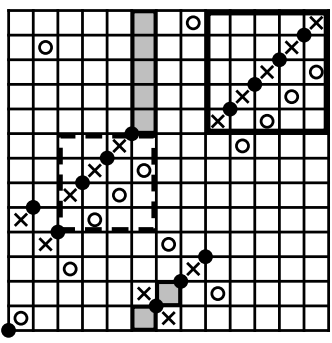

(a) $y_{1}$

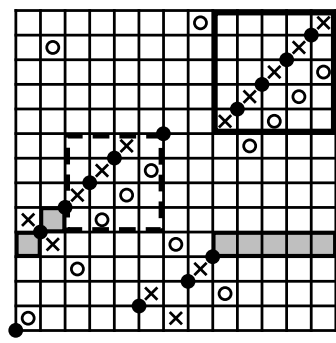

(b) $y_{2}$

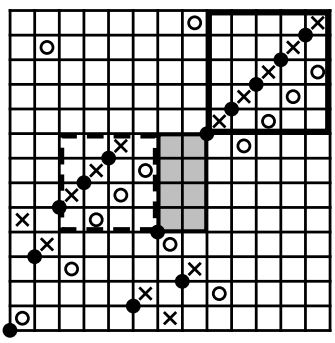

(c) $y_{3}$

Figure 12: Three grid states of $G_{1}(a, b)$. The shaded boxes indicate terms contributing to the differentials of these states.

Proof Write $e=2 b+7$ and $f=2 a+2 b+9$; then the state $x^{+}$is the permutation $(1,6,5,7,8, \ldots, e, 3,2,4, e+1, \ldots, f)=\left(1,6,5,(\ldots)_{1}, e, 3,2,4,(\ldots)_{2}\right)$, where $(\ldots)_{1}=7, \ldots, e-1$ and $(\ldots)_{2}=e+1, \ldots, f$. Define three further states

$$
\begin{aligned}
& y_{1}=\left(1,6,5,(\ldots)_{1}, e, 2,3,4,(\ldots)_{2}\right) \\
& y_{2}=\left(1,5,6,(\ldots)_{1}, 2, e, 3,4,(\ldots)_{2}\right) \\
& y_{3}=\left(1,4,6,(\ldots)_{1}, 2,5,3, e,(\ldots)_{2}\right) .
\end{aligned}
$$

It is then easy to check (Figure 12) that

$$
\begin{aligned}
& \partial\left(y_{1}\right)=\left(1,6,5,(\ldots)_{1}, e, 3,2,4,(\ldots)_{2}\right)+\left(1,6,5,(\ldots)_{1}, 2, e, 3,4,(\ldots)_{2}\right) \\
& \partial\left(y_{2}\right)=\left(1,6,5,(\ldots)_{1}, 2, e, 3,4,(\ldots)_{2}\right)+\left(1,4,6,(\ldots)_{1}, 2, e, 3,5,(\ldots)_{2}\right) \\
& \partial\left(y_{3}\right)=\left(1,4,6,(\ldots)_{1}, 2, e, 3,5,(\ldots)_{2}\right),
\end{aligned}
$$

and so

$$
\partial\left(y_{1}+y_{2}+y_{3}\right)=\left(1,6,5,(\ldots)_{1}, e, 3,2,4,(\ldots)_{2}\right)=x^{+} .
$$

Thus $x^{+}$is null-homologous and $\tilde{\theta}\left(T_{1}(a, b)\right)=0$.

Proposition 3.4 We have $\tilde{\theta}\left(T_{2}(a, b)\right) \neq 0$ and hence $\hat{\theta}\left(T_{2}(a, b)\right) \neq 0$.

Proof We will only need to consider states of the form

$$
(\pi(1), \pi(2), \pi(3), \pi(4), \pi(5), 6,7,8, \ldots, 2 a+2 b+11),
$$

which we abbreviate as $\pi(1), \pi(2), \pi(3), \pi(4), \pi(5)$. In this notation, $x^{+}$for $G_{2}(a, b)$ is $1,5,4,3,2$. To determine whether $x^{+}$is null-homologous, we work as in [16]. 
Let $\mathfrak{A}_{0}=\varnothing$ and $\mathfrak{B}_{0}=\left\{x^{+}\right\}$, and inductively define $\mathfrak{A}_{k}, \mathfrak{B}_{k}$ as follows: let

$$
\begin{aligned}
\mathfrak{A}_{k} & =\left\{\text { states } x \notin \mathfrak{A}_{k-1} \mid y \rightarrow x \text { for some } x \in \mathfrak{B}_{k-1}\right\} \\
\mathfrak{B}_{k} & =\left\{\text { states } y \notin \mathfrak{B}_{k-1} \mid y \rightarrow x \text { for some } x \in \mathfrak{A}_{k}\right\} .
\end{aligned}
$$

This process terminates at some point; let $A, B$ be the free vector spaces over $\mathbb{Z} / 2$ generated by elements of all $\mathfrak{A}_{k}, \mathfrak{B}_{k}$ respectively, and let $C^{\prime}=A \oplus B$. Then the differential $\partial$ on $\widetilde{C F K}$ induces a map $D: A \rightarrow B$, and $x^{+}$is null-homologous in $\widetilde{C F K}$ if and only if $x^{+} \in B$ is in the image of $D$.

For the grid $G_{2}(a, b)$, we successively calculate the $\mathfrak{A}_{k}$ and $\mathfrak{B}_{k}$ as follows; see the appendix for the relevant pictures.

$$
\begin{aligned}
& \left.x^{+}=1,5,4,3,2\right\} \mathfrak{B}_{0}=\left\{x^{+}\right\} \\
& \left.\begin{array}{l}
y_{1}=1,4,5,3,2 \quad y_{1} \rightarrow x^{+} \\
y_{2}=1,5,3,4,2 \quad y_{2} \rightarrow x^{+} \\
y_{3}=1,5,4,2,3 \quad y_{3} \rightarrow x^{+}
\end{array}\right\} \mathfrak{A}_{1}=\left\{y_{1}, y_{2}, y_{3}\right\} \\
& \left.\begin{array}{l}
x_{1}=4,1,5,3,2 \quad x_{1} \leftarrow y_{1} \\
x_{2}=3,5,1,4,2 \quad x_{2} \leftarrow y_{2} \\
x_{3}=2,5,4,1,3 \quad x_{3} \leftarrow y_{3}
\end{array}\right\} \mathfrak{B}_{1}=\left\{x_{1}, x_{2}, x_{3}\right\} \\
& y_{4}=4,1,5,2,3 \quad y_{4} \rightarrow x_{1} \\
& \left.\begin{array}{l}
y_{5}=3,5,1,2,4 \quad y_{5} \rightarrow x_{2} \\
y_{6}=2,4,5,1,3 \quad y_{6} \rightarrow x_{3}
\end{array}\right\} \mathfrak{A}_{2}=\left\{y_{4}, y_{5}, y_{6}, y_{7}\right\} \\
& \left.y_{7}=2,5,3,1,4 \quad y_{7} \rightarrow x_{3}\right) \\
& x_{4}=4,2,5,1,3 \quad x_{4} \leftarrow y_{4}, y_{6} \\
& \left.\begin{array}{l}
x_{5}=3,5,2,1,4 x_{5} \leftarrow y_{5}, y_{7} \\
x_{6}=4,1,2,5,3 \quad x_{6} \leftarrow y_{4}
\end{array}\right\} \mathfrak{B}_{2}=\left\{x_{4}, x_{5}, x_{6}, x_{7}\right\} \\
& x_{7}=2,4,1,5,3 \quad x_{7} \leftarrow y_{6} \\
& y_{8}=1,4,2,5,3 \quad y_{8} \rightarrow x_{6}, x_{7} \\
& \left.\begin{array}{rl}
y_{9} & =4,1,2,3,5 \quad y_{9} \rightarrow x_{6} \\
y_{10} & =5,1,2,4,3 \quad y_{10} \rightarrow x_{6} \\
y_{11} & =2,4,1,3,5 \quad y_{11} \rightarrow x_{7}
\end{array}\right\} \mathfrak{A}_{3}=\left\{y_{8}, y_{9}, y_{10}, y_{11}\right\} \\
& \left.\begin{array}{l}
x_{8}=4,2,1,3,5 \quad x_{8} \leftarrow y_{9}, y_{11} \\
x_{9}=5,2,1,4,3 \quad x_{9} \leftarrow y_{10}
\end{array}\right\} \mathfrak{B}_{3}=\left\{x_{8}, x_{9}\right\} \\
& \left.y_{12}=5,2,1,3,4 \quad y_{12} \rightarrow x_{8}, x_{9}\right\} \mathfrak{A}_{4}=\left\{y_{12}\right\} \text {. }
\end{aligned}
$$

We have $\mathfrak{B}_{4}=\mathfrak{A}_{5}=\varnothing$ and the process terminates here. 
The subcomplex $C$ is generated by $x^{+}, x_{1}, \ldots, x_{9}, y_{1}, \ldots, y_{12}$. Below is the matrix for the adjoint (transpose) of the map $D: A \rightarrow B$, with rows corresponding to $x^{+}, x_{1}, \ldots, x_{9}$ and columns corresponding to $y_{1}, \ldots, y_{12}$, along with its row reduction:

$$
\left(\begin{array}{llllllllll}
1 & 1 & 0 & 0 & 0 & 0 & 0 & 0 & 0 & 0 \\
1 & 0 & 1 & 0 & 0 & 0 & 0 & 0 & 0 & 0 \\
1 & 0 & 0 & 1 & 0 & 0 & 0 & 0 & 0 & 0 \\
0 & 1 & 0 & 0 & 1 & 0 & 1 & 0 & 0 & 0 \\
0 & 0 & 1 & 0 & 0 & 1 & 0 & 0 & 0 & 0 \\
0 & 0 & 0 & 1 & 1 & 0 & 0 & 1 & 0 & 0 \\
0 & 0 & 0 & 1 & 0 & 1 & 0 & 0 & 0 & 0 \\
0 & 0 & 0 & 0 & 0 & 0 & 1 & 1 & 0 & 0 \\
0 & 0 & 0 & 0 & 0 & 0 & 1 & 0 & 1 & 0 \\
0 & 0 & 0 & 0 & 0 & 0 & 1 & 0 & 0 & 1 \\
0 & 0 & 0 & 0 & 0 & 0 & 0 & 1 & 1 & 0 \\
0 & 0 & 0 & 0 & 0 & 0 & 0 & 0 & 1 & 1
\end{array}\right) \stackrel{\text { row reduce }}{\longrightarrow}\left(\begin{array}{llllllllll}
1 & 0 & 0 & 0 & 0 & 1 & 0 & 0 & 0 & 0 \\
0 & 1 & 0 & 0 & 0 & 1 & 0 & 0 & 0 & 0 \\
0 & 0 & 1 & 0 & 0 & 1 & 0 & 0 & 0 & 0 \\
0 & 0 & 0 & 1 & 0 & 1 & 0 & 0 & 0 & 0 \\
0 & 0 & 0 & 0 & 1 & 1 & 0 & 0 & 0 & 1 \\
0 & 0 & 0 & 0 & 0 & 0 & 1 & 0 & 0 & 1 \\
0 & 0 & 0 & 0 & 0 & 0 & 0 & 1 & 0 & 1 \\
0 & 0 & 0 & 0 & 0 & 0 & 0 & 0 & 1 & 1 \\
0 & 0 & 0 & 0 & 0 & 0 & 0 & 0 & 0 & 0 \\
0 & 0 & 0 & 0 & 0 & 0 & 0 & 0 & 0 & 0 \\
0 & 0 & 0 & 0 & 0 & 0 & 0 & 0 & 0 & 0 \\
0 & 0 & 0 & 0 & 0 & 0 & 0 & 0 & 0 & 0
\end{array}\right) .
$$

We see that $[1,0,0,0,0,0,0,0,0,0]$ is not in the row space of the row-reduced matrix, and hence $x^{+}$is not in the image of $D$. We conclude that $\tilde{\theta}\left(T_{2}(a, b)\right)$ is not nullhomologous.

Propositions 3.3 and 3.4 show that $T_{1}(a, b)$ and $T_{2}(a, b)$ are different as transverse knots, and Theorem 1.1 follows.

\section{Appendix: Grid states for $G_{2}(a, b)$}

On the next two pages, we depict the grid states $x_{i}, y_{i}$ from the proof of Proposition 3.4. For each state, the rectangles comprising the differential for the $y$ states, or the adjoint differential for the $x$ states, are shaded, with darker shading for rectangles from previous states and lighter shading for the others. Some rectangles overlap.

\section{References}

[1] J S Birman, W W Menasco, Stabilization in the braid groups. II. Transversal simplicity of knots, Geom. Topol. 10 (2006) 1425-1452 MR2255503

[2] J S Birman, W W Menasco, A note on closed 3-braids, Commun. Contemp. Math. 10 (2008) 1033-1047 MR2468377

[3] J S Birman, N C Wrinkle, On transversally simple knots, J. Differential Geom. 55 (2000) 325-354 MR1847313 


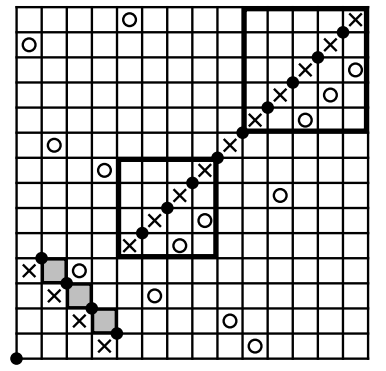

(a) $x^{+}$

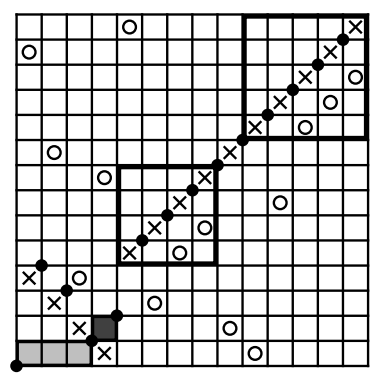

(d) $y_{3}$

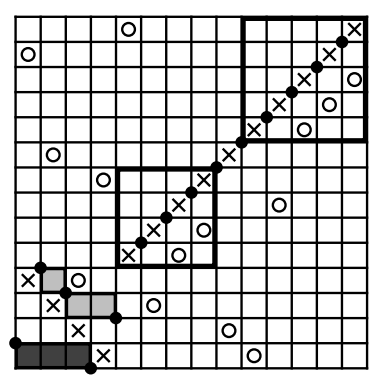

(g) $x_{3}$

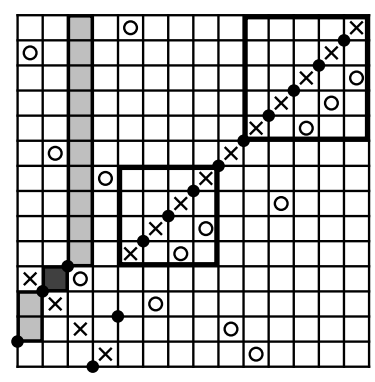

(j) $y_{6}$

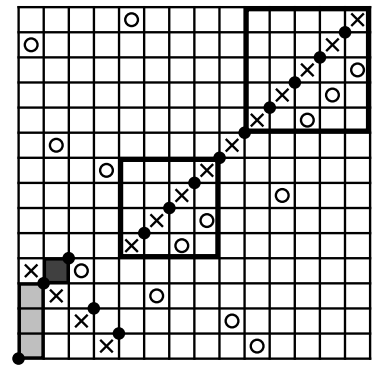

(b) $y_{1}$

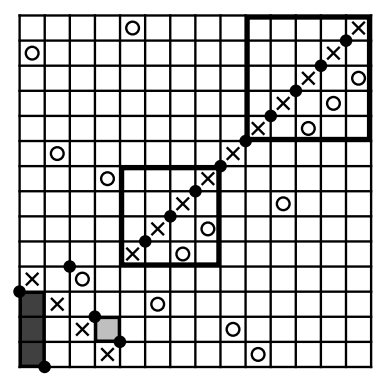

(e) $x_{1}$

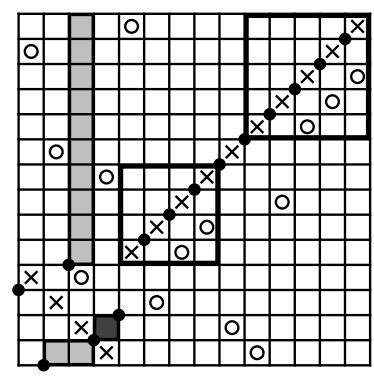

(h) $y_{4}$

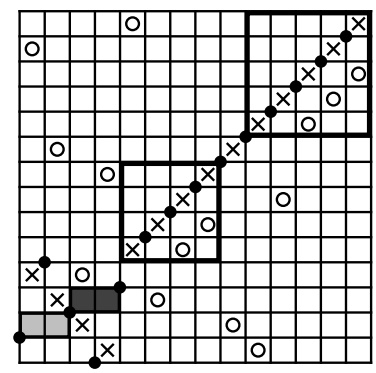

(k) $y_{7}$

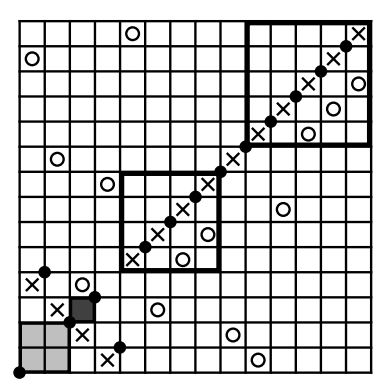

(c) $y_{2}$

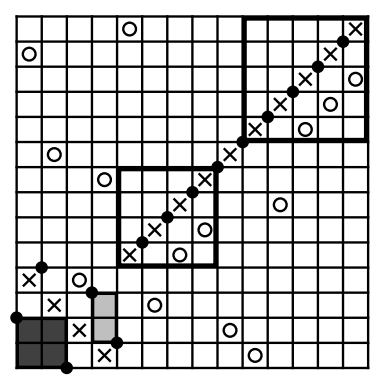

(f) $x_{2}$

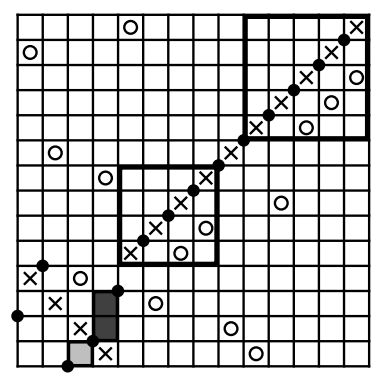

(i) $y_{5}$

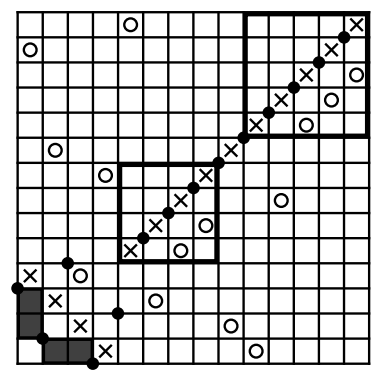

(l) $x_{4}$ 


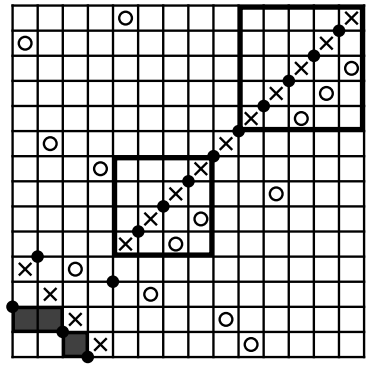

(m) $x_{5}$

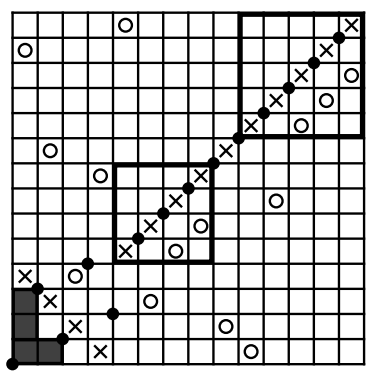

(p) $y_{8}$

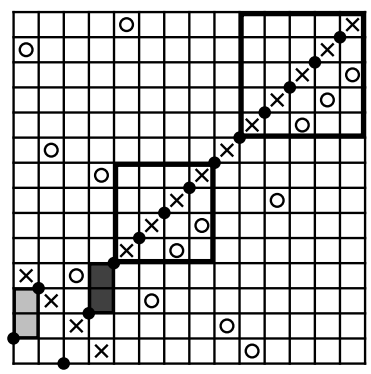

(s) $y_{11}$

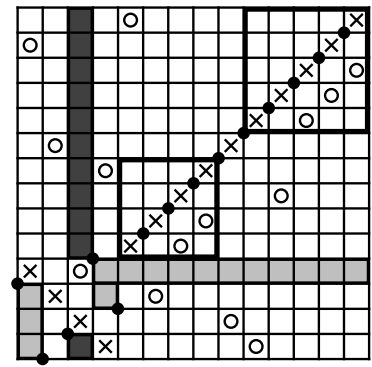

(n) $x_{6}$

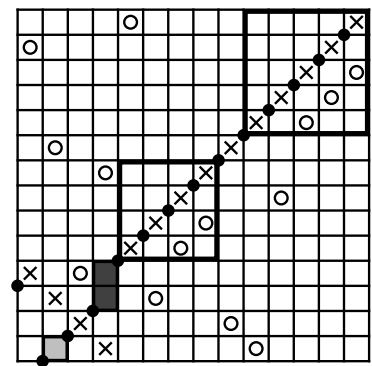

(q) $y_{9}$

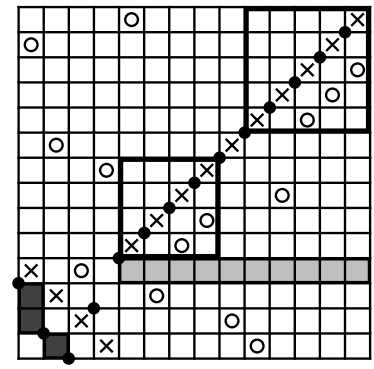

(t) $x_{8}$

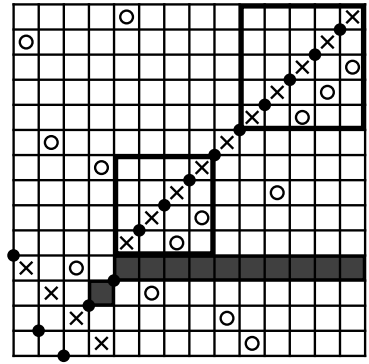

(v) $y_{12}$

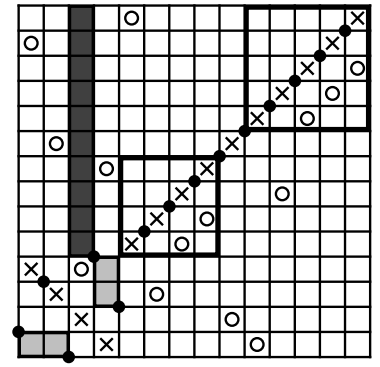

(o) $x_{7}$

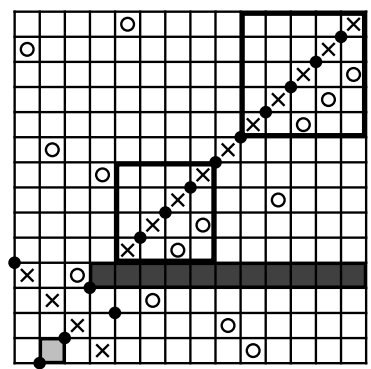

(r) $y_{10}$

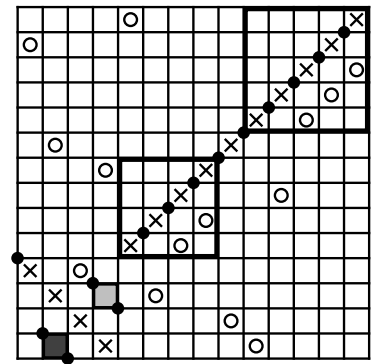

(u) $x_{9}$

Figure 14: Grid states for $G_{2}(a, b)$; here $(a, b)=(1,1)$. 
[4] P R Cromwell, Embedding knots and links in an open book. I. Basic properties, Topology Appl. 64 (1995) 37-58 MR1339757

[5] I A Dynnikov, Arc-presentations of links: monotonic simplification, Fund. Math. 190 (2006) 29-76 MR2232855

[6] Y Eliashberg, Legendrian and transversal knots in tight contact 3-manifolds, from: "Topological methods in modern mathematics (Stony Brook, NY, 1991)", (L R Goldberg, A V Phillips, editors), Publish or Perish, Houston, TX (1993) 171-193 MR1215964

[7] J Epstein, D Fuchs, M Meyer, Chekanov-Eliashberg invariants and transverse approximations of Legendrian knots, Pacific J. Math. 201 (2001) 89-106 MR1867893

[8] J B Etnyre, Transversal torus knots, Geom. Topol. 3 (1999) 253-268 MR1714912

[9] J B Etnyre, Legendrian and transversal knots, from: "Handbook of knot theory", (W Menasco, M Thistlethwaite, editors), Elsevier, Amsterdam (2005) 105-185 MR2179261

[10] J B Etnyre, K Honda, Knots and contact geometry. I. Torus knots and the figure eight knot, J. Symplectic Geom. 1 (2001) 63-120 MR1959579

[11] J B Etnyre, K Honda, Cabling and transverse simplicity, Ann. of Math. (2) 162 (2005) 1305-1333 MR2179731

[12] J Hoste, M Thistlethwaite, Knotscape Available at http://www.math.utk.edu/ $\sim$ morwen/knotscape.html

[13] T Kálmán, Braid-positive Legendrian links, Int. Math. Res. Not. (2006) Art ID 14874, 29 MR2272097

[14] K Kawamuro, Connect sum and transversely non simple knots, Math. Proc. Cambridge Philos. Soc. 146 (2009) 661-669 MR2496350

[15] C Manolescu, P Ozsváth, S Sarkar, A combinatorial description of knot Floer homology, Ann. of Math. (2) 169 (2009) 633-660 MR2480614

[16] L Ng, P Ozsváth, D Thurston, Transverse knots distinguished by knot Floer homology, J. Symplectic Geom. 6 (2008) 461-490 MR2471100

[17] L Ng, L Traynor, Legendrian solid-torus links, J. Symplectic Geom. 2 (2004) 411-443 MR2131643

[18] S Y Orevkov, V V Shevchishin, Markov theorem for transversal links, J. Knot Theory Ramifications 12 (2003) 905-913 MR2017961

[19] P Ozsváth, J Rasmussen, Z Szabó, Odd Khovanov homology arXiv:0710.4300

[20] P Ozsváth, A Stipsicz, Contact surgeries and the transverse invariant in knot Floer homology arXiv:0803.1252

[21] P Ozsváth, Z Szabó, Holomorphic disks and knot invariants, Adv. Math. 186 (2004) 58-116 MR2065507 
[22] P Ozsváth, Z Szabó, D Thurston, Legendrian knots, transverse knots and combinatorial Floer homology, Geom. Topol. 12 (2008) 941-980 MR2403802

[23] J A Rasmussen, Floer homology and knot complements, PhD thesis, Harvard University (2003) arXiv:math.GT/0204056

[24] V Vértesi, Transversely nonsimple knots, Algebr. Geom. Topol. 8 (2008) 1481-1498 MR2443251

[25] N C Wrinkle, The Markov Theorem for transverse knots arXiv:math.GT/0202055

Department of Mathematics, Massachusetts Institute of Technology

Cambridge, MA 02139, USA

Mathematics Department, Duke University

Durham, NC 27708, USA

tirasan@math.mit.edu, ng@math.duke.edu

Received: 4 February 2009 Revised: 5 June 2009 